\title{
Enhancement of pulmonary vascular remodelling and inflammatory genes with VIP gene deletion
}

\author{
S.A. Hamidi*,\#, S. Prabhakar" and S.I. Said*,\#
}

ABSTRACT: The pathogenesis of idiopathic pulmonary arterial hypertension (PAH) remains poorly understood. The present authors recently reported that mice with vasoactive intestinal peptide (VIP) gene disruption show a spontaneous phenotype of PAH, with pulmonary vascular remodelling and lung inflammation.

To explore the underlying molecular mechanisms in this model, it was examined whether absence of the VIP gene might alter the expression of additional genes involved in the pathogenesis of $\mathrm{PAH}$, as single-gene deletions, in the absence of hypoxia, rarely result in significant pulmonary vascular remodelling.

Lung tissue from mice with targeted disruption of the vasoactive intestinal peptide gene (VIP $/ /$ mice) and from control mice was subjected to whole-genome gene microarray analysis, and the results validated with quantitative, real-time PCR. Lungs from $\mathrm{VIP}^{-/-}$mice showed a wide range of significant gene expression alterations, including overexpression of genes that promote pulmonary vascular smooth muscle cell proliferation, underexpression of antiproliferative genes and upregulation of pro-inflammatory genes.

In conclusion, vasoactive intestinal peptide is a pivotal modulator of genes controlling the pulmonary vasculature, its deficiency alone resulting in gene expression alterations that can readily explain both the vascular remodelling and associated inflammatory response in pulmonary arterial hypertension. The present findings shed more light on the molecular mechanisms of pulmonary arterial hypertension, and could lead to better understanding of the pathogenesis of human pulmonary arterial hypertension, and hence to improved therapy.

KEYWORDS: Gene expression and regulation, pulmonary hypertension, quantitative PCR, right heart failure

$\mathrm{T}$ he present authors recently described a model of pulmonary hypertension in airbreathing, nonhypoxaemic mice with targeted disruption of the vasoactive intestinal peptide (VIP) gene (VIP ${ }^{-/-}$mice) [1]. The pulmonary vascular abnormalities in these mice, notably pulmonary vascular smooth muscle and collagen proliferation, and right ventricular (RV) hypertrophy are reminiscent of those seen in idiopathic pulmonary arterial hypertension (IPAH). There was also evidence of lung inflammation, which may contribute to the pathogenesis of IPAH. Both the vascular remodelling and inflammation were markedly attenuated by VIP replacement [1]. This mouse model, however, differs from the human disease in several respects: 1) pulmonary hypertension is of a moderate degree; 2) despite often pronounced medial thickening, intimal proliferation and plexiform lesions are not observed; and 3) in contrast to the clinical condition, the mouse phenotype is more prominently expressed in males than in females.

The purpose of the present study was to uncover the molecular mechanisms by which disruption of the VIP gene could lead to expression of pulmonary arterial hypertension (PAH), pulmonary vascular remodelling, RV hypertrophy and lung inflammation. VIP has long been identified as a pulmonary, as well as systemic, vasodilator, and is also known to inhibit human pulmonary arterial smooth muscle cell (PASMC) proliferation. Its absence, therefore, might be expected to
AFFILIATIONS

*Pulmonary and Critical Care Medicine, State University of New York, Stony Brook,

"Dept of Veterans Affairs Medical Center, Northport, NY, and

- SuperArray Bioscience Corporation, Fredrick, MD, USA

CORRESPONDENCE

S.I. Said

Pulmonary and Critical Care Medicine State University of New York Health Sciences Center

T-17-040

Stony Brook

NY 11784-8172

USA

Fax: 16314447502

E-mail: sami.i.said@stonybrook.edu

Received

August 132007

Accepted after revision:

September 272007

SUPPORT STATEMENT

The research was supported by National Institutes of Health (Bethesda, MA, USA) grants HL70212 and HL-68188 (to S.I. Said) and by the Dept of Veterans Affairs, Washington, DC, USA.

STATMENT OF INTEREST None declared. 
predispose to the cardiovascular pathology of pulmonary hypertension. To the present authors' knowledge, however, no other single-gene deletion, especially in the absence of the added stimulus of chronic hypoxia, has been reported to result in $\mathrm{PAH}$ with significant pulmonary vascular remodelling. Therefore, it is hypothesised that loss of the VIP gene might cause major alterations in the expression of multiple genes and pathways that could explain the development of the PAH phenotype. To test this hypothesis, lung tissue from $\mathrm{VIP}^{-/-}$and wild-type (WT) mice was subjected to whole-genome gene microarray analysis, and the microarray results validated using quantitative, real-time PCR, with special attention to genes and pathways known to either promote or modulate pulmonary vascular remodelling. The current approach has been advocated as likely to provide insights into the complex pathogenesis of the disease [2].

\section{MATERIALS AND METHODS}

\section{Animals}

$\mathrm{VIP}^{-/-}$and control C57BL/6 WT mice were as described [1]. The entire study was approved by the present authors' institutional animal review committees and was performed according to the Helsinki Convention for the use and care of animals.

\section{Agilent whole-genome microarray analysis}

Lungs from five male VIP knockout (KO) and five male WT mice, 20-24-weeks old, were removed from freshly euthanised animals, immersed in RNAlater ${ }^{\mathrm{TM}}$ (10 mL per $1 \mathrm{mg}$ of tissue; Ambion, Austin, TX, USA), fresh-frozen in liquid nitrogen and shipped overnight on dry ice to Superarray Biosciences (Fredrick, MD, USA). Microarray data were collected at Superarray Biosciences, using the Whole Mouse Genome Oligo Microarray Kit with SurePrint technology $(4 \times 44 \mathrm{~K}$ slide format; Agilent Technologies, Palo Alto, CA, USA).

\section{Real-time PCR}

The microarray results were validated using quantitative realtime PCR. RNA was examined from the same lungs subjected to microarray analysis, i.e. from five $\mathrm{KO}$ and five WT mice. The procedures were carried out and the results were analysed at Superarray Biosciences. Gene expression data from $\mathrm{VIP}^{-/-}$mice were quantified relative to data from WT mice, according to the $2^{-\Delta \Delta C} \mathrm{~T}$ method [3]. Differences in fold-change for each gene were compared pairwise by the two-tailed t-test. Details of the aforementioned methods can be found in the online supplementary material.

\section{RESULTS}

\section{Gene expression alterations}

Data obtained from the whole-genome microarray analysis was filtered with respect to the genes involved in pulmonary vascular remodelling. All the changes in gene expression obtained by microarray analysis were validated by quantitative real-time PCR using the lungs of five $\mathrm{VIP}^{-/-}$and five WT mice (table 1). The results show a wide range of significant gene expression alterations in $\mathrm{VIP}^{-/-}$mice relative to WT mice. The alterations fell into three categories, known collectively to contribute to the PAH phenotype: 1) overexpression of several key genes associated with pulmonary vasoconstriction and pulmonary vascular remodelling; 2) underexpression of dominant vasodilator, antiproliferative genes; and 3) upregulation of inflammatory genes (table 1).

\section{Vasoconstrictor pro-remodelling genes}

The expression of genes associated with vascular smooth muscle cell contraction [4,5], including myosin light polypeptides 1 and 3 (Myl1 and Myl3, with 16.2- and 32.6-fold change, respectively) and myosin heavy polypeptides 1, 4 and 8 (Myh1, Myh4 and Myh8, with 331.5-, 294.3- and 37.3-fold change, respectively) were highly and significantly induced in the $\mathrm{VIP}^{-/-}$mice, as compared with the WT mice. Genes associated with smooth muscle cell migration, proliferation and collagen deposition, including Rho guanosine diphosphate dissociation inhibitor $\beta$ (Arhgdib, 1.7-fold change), angiopoietin 1 (Angpt1, 1.7-fold change) [6] and pro-collagen type V alpha1 (Col5a1, 1.3-fold change) [7], were also upregulated slightly but significantly.

\section{Vasodilator antiremodelling genes}

Conversely, genes that constitute the principal lines of defence against $\mathrm{PAH}$ and pulmonary vascular remodelling were underexpressed relative to WT mice. Together with the VIP gene (0.01), other genes included: $\beta_{2}$-adrenergic receptor (Adrb2, 0.3); bone morphogenetic protein 2 (BMP2, 0.5), Smad1 (0.7); guanosine triphosphate (GTP) cyclohydrolase 1 (Gch1, 0.6), the rate-limiting enzyme in the biosynthesis of tetrahydrobiopterin (BH4) [8], an essential co-factor for the activity of endothelial nitric oxide (NO) synthase (eNOS or NOS3); NOS3 itself (0.5), a pivotal regulator of vascular integrity [9]; prostacyclin synthase (Ptgis, 0.6) [10]; and vascular endothelial growth factor $C(V e g f c, 0.6)$ [11]. Other related underexpressed genes included: apolipoprotein $\mathrm{E}$ (Apoe, 0.5), which neutralises platelet-derived growth factor signalling [12], both of which are also deficient in IPAH patients.

\section{Inflammatory genes}

Genes of several inflammatory cytokines and chemokines, often linked to vascular remodelling [13, 14], were upregulated. These included: members of the tumour necrosis factor (TNF) receptor superfamily (Tnfrsf13c and Tnfrsf19, 2.4- and 2.2-fold change); C-C motif chemokine receptors (Ccr6, 4.6-fold change); and mast cell protease 8 (Mcpt8, 6.8-fold change), which also has angiotensin-forming activity [15]. These gene expression alterations are supported by measurements of inflammatory cytokines, chemokines, immunoglobulin E and proteases in bronchoalveolar lavage fluid, and cellular infiltrates in lung sections [16].

Additional gene expression changes noted on microarray analysis but not yet confirmed by real-time PCR included the following. 1) From the vasoconstrictor pro-remodelling genes group: upregulation of Rho GTPase-activating proteins (Arhgap) [17]; endothelin receptor A (Ednra) [18]; plateletderived growth factor $\beta$ polypeptide $(P d g f b)$; and plateletderived growth factor receptor $\beta$ polypeptide (Pdgfrb) [19]. 2) From the vasodilator antiremodelling genes group: downregulation of potassium voltage-gated channels [20]. 3) From the inflammatory genes group: upregulation of interleukin (IL)-1a, IL-1b and IL-6, and nuclear factor of activated T-cells (Nfatc2). Failure to confirm these changes by real-time PCR 


\begin{tabular}{|c|c|c|c|c|c|c|c|}
\hline $\begin{array}{l}\text { TABLE } 1 \text { Gene expressi } \\
\text { type (WT) mice }\end{array}$ & n data ir & ce with & ruption & active i & al pep & \% mice & e to wild- \\
\hline \multirow[t]{2}{*}{ Gene } & \multirow[t]{2}{*}{ Symbol } & \multicolumn{3}{|c|}{ Microarray } & \multicolumn{3}{|c|}{ Real-time PCR } \\
\hline & & Change & $\mathbf{V I P}^{-/-} / \mathbf{W T}$ & p-value & Change & $\mathbf{V I P}^{-/-} / \mathbf{W T}$ & p-value \\
\hline \multicolumn{8}{|l|}{$\begin{array}{l}\text { Vasoconstrictor pro-remodel- } \\
\text { ling genes }\end{array}$} \\
\hline Myosin heavy polypeptide 4 & Myh4 & $\uparrow$ & 29.4 & 0.0052 & $\uparrow$ & 294.3 & 0.0007 \\
\hline Myosin heavy polypeptide 8 & Myh8 & $\uparrow$ & 44.3 & 0.0320 & $\uparrow$ & 37.3 & 0.0020 \\
\hline Myosin light polypeptide 3 & Myl3 & $\uparrow$ & 30.1 & 0.0051 & $\uparrow$ & 32.6 & 0.0011 \\
\hline Myosin light polypeptide 1 & Myl1 & $\uparrow$ & 21.2 & 0.0003 & $\uparrow$ & 16.2 & 0.0005 \\
\hline Angiopoetin 1 & Angpt1 & NC & 1.0 & 0.8864 & $\uparrow$ & 1.6 & 0.0262 \\
\hline $\begin{array}{l}\text { Vasoactive intestinal polypep- } \\
\text { tide }\end{array}$ & Vip & $\downarrow$ & 0.04 & 0.0012 & $\downarrow$ & 0.01 & 0.0000 \\
\hline$\beta_{2}$-Adrenergic receptor & Adrb2 & $\downarrow$ & 0.4 & 0.0392 & $\downarrow$ & 0.3 & 0.0006 \\
\hline Apolipoprotein E & Apoe & $\downarrow$ & 0.8 & 0.0112 & $\downarrow$ & 0.5 & 0.0043 \\
\hline Bone morphogenetic protein 2 & Bmp2 & $\downarrow$ & 0.9 & 0.0740 & $\downarrow$ & 0.5 & 0.0099 \\
\hline NO synthase 3 endothelial cell & Nos3 & $\downarrow$ & 0.6 & 0.0169 & $\downarrow$ & 0.5 & 0.0189 \\
\hline GTP cyclohydrolase 1 & Gch1 & $\downarrow$ & 0.8 & 0.0295 & $\downarrow$ & 0.6 & 0.0056 \\
\hline Prostaglandin $12^{+}$synthase & Ptgis & $\downarrow$ & 0.8 & 0.1020 & $\downarrow$ & 0.6 & 0.0050 \\
\hline $\begin{array}{l}\text { Vascular endothelial growth } \\
\text { factor } C\end{array}$ & Vegfc & $\downarrow$ & 0.6 & 0.0465 & $\downarrow$ & 0.6 & 0.0217 \\
\hline MAD homolog $1^{\S}$ & Smad1 & $\downarrow$ & 0.8 & 0.0208 & $\downarrow$ & 0.7 & 0.0670 \\
\hline
\end{tabular}

GDI: guanine nucleoticle dissociation inhibitor; NO: nitric oxide; GTP: guanosine triphosphate; TNF: tumour necrosis factor. \#: changes in gene expression, first noted in microarray analysis, were later confirmed by real-time PCR. The degree of overexpression is indicated by $\mathrm{x}$-fold change, underexpression as a fraction of that in WT

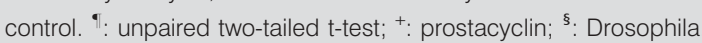

may be because the pathway alterations occur at the translational level, or additional stimuli, such as hypoxia, are needed in addition to loss of VIP gene to elicit the changes at the gene level.

\section{DISCUSSION}

The results clearly show that disruption of the VIP gene elicits a dramatic set of significant gene expression alterations, relative to WT mice. These alterations comprise the three groups known collectively to be responsible for the PAH phenotype, namely: 1) overexpression of genes associated with pulmonary vasoconstriction and pulmonary vascular remodelling; 2) underexpression of vasodilator, antiproliferative genes; and 3) upregulation of inflammatory genes.

As the whole lung rather than microdissected pulmonary arteries were examined, it is possible that the changes in gene expression reflected both vascular and nonvascular alterations in $\mathrm{VIP}^{-/-}$mice. Nevertheless, the striking similarities between gene expression changes in $\mathrm{VIP}^{-/-}$mice and those reported in $\mathrm{IPAH}$ (and corresponding proteomic measurements), further validate the $\mathrm{VIP}^{-/-}$mouse model as one that is closely related to the human disease, and is therefore suitable for exploring the complex mechanisms underlying this disorder.

From the present results, VIP emerges as a dominant modulator of pulmonary vascular remodelling, its absence alone being sufficient to cause such extensive gene expression alterations as to result in a reasonable facsimile of the $\mathrm{PAH}$ phenotype: RV systolic pressure $29.5 \pm 1.0$ versus $16.3 \pm 2.2 \mathrm{cmH}_{2} \mathrm{O}(\mathrm{p}<0.001)$, with RV hypertrophy and considerable pulmonary vascular smooth muscle proliferation [1]. VIP exerts many of the actions highlighted by deletion of its gene, including: 1) increased generation of cyclic adenosine monophosphate (cAMP) and resultant protein kinase (PK)A activation; 2) enhancement of NOS3 activity and NO production via activation of $\mathrm{BH} 4$ [21], and hence of guanylate cyclase activity; 3) neutralisation of the contractile effect of endothelin on pulmonary arterial segments and abrogation of the heightened expression of lung endothelin receptors $A$ and $B$ during hypoxia [22]; 4) suppression of PASMC proliferation by 
direct action in vitro [23]; and 5) inhibition of smooth muscle cell migration via cAMP-dependent PKA activation of Apoe. Thus, largely through its combined activation of the adenylate cyclase/cAMP and guanylate cyclase/cyclic guanosine monophosphate pathways, VIP normally creates a set of complementary and powerful antiremodelling defence mechanisms in pulmonary vessels.

In addition to its vascular effects, VIP has a number of important anti-inflammatory effects, including: 1) modulation of T-cell proliferation and activation; 2) inhibition of nuclear factor- $\kappa \mathrm{B}$ activation; 3) suppression of pro-inflammatory cytokines TNF- $\alpha$, IL-6, IL-12, IL-18, chemokine regulated on activation, normal T-cell expressed and secreted (RANTES), and inducible NOS; and 4) upregulation of anti-inflammatory IL-10 [24].

The inflammatory component of $\mathrm{PAH}$ pathology in $\mathrm{VIP}^{-/-}$mice parallels that observed in human $\mathrm{PAH}$. Convincing evidence exists for various manifestations of lung inflammation in $\mathrm{PAH}$, particularly in certain forms of the disorder, but the possible relationship of inflammation to the vascular remodelling remains uncertain $[13,14]$. The co-existence of inflammation and vascular lesions in the present experimental model suggests that both pathological processes could have a common pathogenetic basis.

Finally, while the present results strongly suggest a pivotal role for the vasoactive intestinal peptide gene as a regulator of both smooth muscle function and inflammation, vasoactive intestinal peptide may share this position of dominance with another "master regulator": the nuclear factor of activated Tcells. This transcription factor can trigger both inflammatory and vascular smooth muscle cell proliferation pathways, and its activation has recently been invoked as the principal mechanism of chronic hypoxia-induced pulmonary arterial remodelling [25] and many of the findings in idiopathic pulmonary hypertension [26]. Therefore, it is tempting to speculate that vasoactive intestinal peptide may exert its broad modulator influence, at least in part, by inhibiting the activation of the nuclear factor of activated T-cells. Evidence for such inhibition, in another context, has been reported previously [27].

\section{REFERENCES}

1 Said SI, Hamidi SA, Dickman KG, et al. Moderate pulmonary arterial hypertension in male mice lacking the vasoactive intestinal peptide gene. Circulation 2007; 115: 1260-1268.

2 Bull TM, Coldren CD, Geraci MW, Voelkel NF. Gene expression profiling in pulmonary hypertension. Proc Am Thorac Soc 2007; 4: 117-120.

3 Livak KJ, Schmittgen TD. Analysis of relative gene expression data using real-time quantitative PCR and the $2^{-\Delta \Delta C}$ T Method. Methods 2001; 25: 402-408.

4 Wada H, Hasegawa K, Morimoto $\mathrm{T}$, et al. CalcineurinGATA-6 pathway is involved in smooth muscle-specific transcription. J Cell Biol 2002; 156: 983-991.

5 Pfitzer G. Invited review: regulation of myosin phosphorylation in smooth muscle. J Appl Physiol 2001; 91: 497-503.
6 Dewachter L, Adnot S, Fadel E, et al. Angiopoietin/Tie2 pathway influences smooth muscle hyperplasia in idiopathic pulmonary hypertension. Am J Respir Crit Care Med 2006; 174: 1025-1033.

7 Edgar AJ, Chacón MR, Bishop AE, Yacoub MH, Polak JM. Upregulated genes in sporadic, idiopathic pulmonary arterial hypertension. Respir Res 2006; 7: 1.

8 Khoo JP, Zhao L, Alp NJ, et al. Pivotal role for endothelial tetrahydrobiopterin in pulmonary hypertension. Circulation 2005; 111: 2126-2133.

9 Giaid A, Saleh D. Reduced expression of endothelial nitric oxide synthase in the lungs of patients with pulmonary hypertension. N Engl J Med 1995; 333: 214-221.

10 Tuder RM, Cool CD, Geraci MW, et al. Prostacyclin synthase expression is decreased in lungs from patients with severe pulmonary hypertension. Am J Respir Crit Care Med 1999; 159: 1925-1932.

11 Voelkel NF, Vandivier RW, Tuder RM. Vascular endothelial growth factor in the lung. Am J Physiol Lung Cell Mol Physiol 2006; 290: L209-L221.

12 Hansmann G, Wagner RA, Schellong S, et al. Pulmonary arterial hypertension is linked to insulin resistance and reversed by peroxisome proliferator-activated receptor- $\gamma$ activation. Circulation 2007; 115: 1275-1284.

13 Dorfmüller $\mathrm{P}$, Perros F, Balabanian K, Humbert M. Inflammation in pulmonary arterial hypertension. Eur Respir J 2003; 22: 358-363.

14 Nicolls MR, Taraseviciene-Stewart L, Rai PR, Badesch DB, Voelkel NF. Autoimmunity and pulmonary hypertension: a perspective. Eur Respir J 2005; 26: 1110-1118.

15 Hamada H, Terai M, Kimura H, Hirano K, Oana S, Niimi H. Increased expression of mast cell chymase in the lungs of patients with congenital heart disease associated with early pulmonary vascular disease. Am J Respir Crit Care Med 1999; 160: 1303-1308.

16 Szema AM, Hamidi SA, Lyubsky S, et al. Mice lacking the VIP gene show airway hyperresponsiveness and airway inflammation, partially reversible by VIP. Am J Physiol Lung Cell Mol Physiol 2006; 291: L880-L886.

17 Oka M, Homma N, Taraseviciene-Stewart L, et al. Rho kinase-mediated vasoconstriction is important in severe occlusive pulmonary arterial hypertension in rats. Circ Res 2007; 100: 923-929.

18 Giaid A, Yanagisawa M, Langleben D, et al. Expression of endothelin-1 in the lungs of patients with pulmonary hypertension. N Engl J Med 1993; 328: 1732-1739.

19 Barst RJ. PDGF signaling in pulmonary arterial hypertension. J Clin Invest 2005; 115: 2691-2694.

20 Yuan JX, Aldinger AM, Juhaszova M, et al. Dysfunctional voltage-gated $\mathrm{K}^{+}$channels in pulmonary artery smooth muscle cells of patients with primary pulmonary hypertension. Circulation 1998; 98: 1400-1406.

21 Anastasiadis PZ, Bezin L, Gordon LJ, et al. Vasoactive intestinal peptide induces both tyrosine hydroxylase activity and tetrahydrobiopterin biosynthesis in PC12 cells. Neuroscience 1998; 86: 179-189.

22 Said SI. Mediators and modulators of pulmonary arterial hypertension. Am J Physiol Lung Cell Mol Physiol 2006; 291: L547-L558.

23 Petkov V, Mosgoeller W, Ziesche R, et al. Vasoactive intestinal peptide as a new drug for treatment of primary 
pulmonary hypertension. J Clin Invest 2003; 111: 1339-1346.

24 Gomariz RP, Martinez C, Abad C, Leceta J, Delgado M. Immunology of VIP: a review and therapeutical perspectives. Curr Pharm Des 2001; 7: 89-111.

25 de Frutos S, Spangler R, Alò D, Bosc LV. NFATc3 mediates chronic hypoxia-induced pulmonary arterial remodeling with $\alpha$-actin up-regulation. J Biol Chem 2007; 282: 1508115089.
26 Bonnet S, Rochefort G, Sutendra G, et al. The nuclear factor of activated $\mathrm{T}$ cells in pulmonary arterial hypertension can be therapeutically targeted. Proc Natl Acad Sci USA 2007; 104: 11418-11423.

27 Delgado M, Ganea D. Vasoactive intestinal peptide and pituitary adenylate cyclase-activating polypeptide inhibit expression of Fas ligand in activated $\mathrm{T}$ lymphocytes by regulating c-Myc, NF- $\mathrm{\kappa B}, \mathrm{NF}-\mathrm{AT}$, and early growth factors 2/3. J Immunol 2001; 166: 1028-1040. 